\title{
ROBOTIC MANUFACTURING EXPERIMENTAL RESEARCH - MACHINING FORCES ANALYSIS FOR AN ALUMINIUM PROFILE MILLING PROCESS
}

\author{
IVAN, A[ndrei] - M[ario]; NICOLESCU, A[drian] F[lorin]; \\ STRAJESCU, E[ugen] R[adu] \& AVRAM, G[eorgia] - C[ezara]
}

\begin{abstract}
The paper deals with some of the aspects of robotic manufacturing in terms of measuring and analyzing milling forces that appear at the tool-part interface during an aluminium profile machining process. In order to achieve the goals of the research project, a dedicated robotic manufacturing cell developed in prevoius works was programmed for a milling application in which several chamfering operations were performed on an aluminium rectangular profile. The machining forces were measured with a special dynamometer integrated into the system, and the obtained data was analyzed in order to determine the behaviour of the robotic system during machining operations. Keywords: robotic machining, robotic manufacturing cell, robotic offline programming, milling forces
\end{abstract}

\section{INTRODUCTION}

It is well known that, in the world of manufacturing, machine-tools have held the dominant position as process equipment for decades. Yet, even today, there are still certain areas in the field in which a significant number of operations are performed by human workers, such as deburring, polishing or chamfering. Also, given the kinematic flexibility issues, complex-shaped parts pose important difficulties for most machine-tools available today on the market.

These aspects generate enough arguments for a number of specialists to concentrate their research on integrating industrial robotic systems in machining processes. Although it is widely accepted that robots will not replace machine-tools in manufacturing (at least, not in the predictable future) [1], many of the research works illustrated in the literature focus on enlarging the scope of robotic systems integrated in manufacturing cells [2]. There are two approaches in this area: finding applications for which the essential characteristics of industrial robots provide certain advantages and finding ways of improving robot performances in order to make them suitable for a wider range of operations [3].

Considering the second approach, there are multiple issues addressed in the literature. Among these are aspects regarding robot system calibration, kinematic error compensation, robotic cell structure and passive / active force control in machining operations [4]. This paper illustrates a part of a research project that aims at finding the different influence factors in robotic machining applications, determining the share of each element in error occurrence and form a suitable basis for proposing efficient methods for improving robot performances in manufacturing processes.

The research was limited to analyzing aspects in the field of applications requiring low machining forces [5], in which general-purpose articulated arm robots, with low to medium payloads, manipulating self-driven tools are used. From this point of view, the influence factors in the machining process were identified and analyzed. One of the sections of the research project was focused on discussing process parameters and machining forces as contributing factors to the success of a manufacturing operation.

Taking the above aspects into consideration, the works presented in this paper were based on the fact that machining forces that occur in a milling process represent a factor that influences the precision of manufacturing. The research was focused on applications requiring low machining power, using flexible cells that integrate articulated arm robot equipped with self-driven tools. Further, the magnitude of the machining forces was determined in an experimental approach for a chamfering operation and the influence on robot's precision during the application was evaluated.

\section{EXPERIMENTAL ROBOTIC MANUFACTURING CELL}

In order to provide an appropiate experimental setup for collecting the necessary data, a robotic manufacturing cell had to be developed. Considering that the research was limited to applications requiring low machining forces, the cell was built according to the first operating principle of robotic manufacturing cells, which specifies the following structural characterisitcs:

a. the part is mounted on a fixed workpost or on a workpiece positioner providing up to two workposts and up to three numerically controlled axes;

b. the machining operations are performed by an articulated arm robot equipped with a low to medium power self-driven tool;

c. the end-effector can be designed for milling-type operations, in which case it integrates a radially compliant system, or for drilling / polishing type operations, in which case it integrates an axially compliant system (thus providing a passive force control method for the application);

d. an automated tool changing system can be optionally included in the robotic manufacturing cell comprising of a tool storage structure and an automated endeffector coupling-decoupling device;

also, the cell can integrate a force / torque measuring device, either mounted at the wrist flange / end-effector interface or between the part and the workpost. 


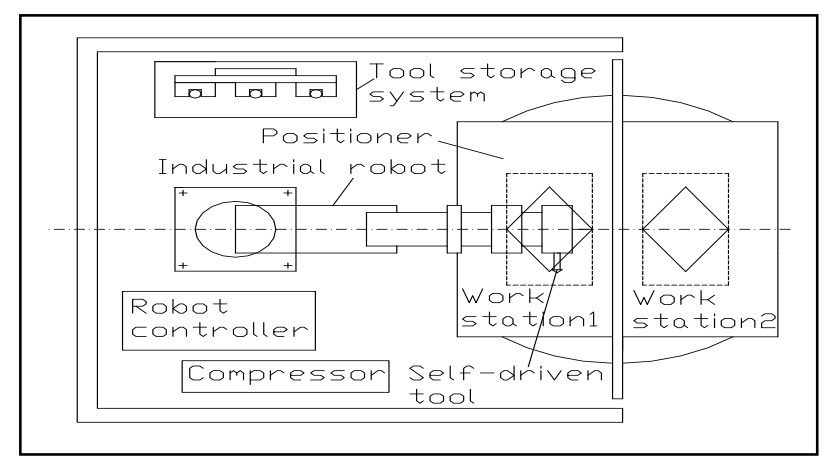

Fig. 1. Typical cell layout for the first operating principle

As it can be noticed, depending on the specific application for which the robotic manufacturing cell is built, the last two main components can be optional. Yet, for the purpose of collecting the necessary experimental data for proposed research goals, a force / torque measuring device was included [6] [7]. The general structure of a robotic manufacturing cell corresponding to the first operating principle is shown in Fig. 1.

Considering the above characteristics corresponding to the first operating principle, the experimental robotic cell was developed accordingly [8]. The structure of the manufacturing cell includes a Kawasaki FS10E 6-axis articulated arm industrial robot with $10 \mathrm{~kg}$ payload, equipped with an ATI RC 340E radially compliant deburring end-effector featuring $40000 \mathrm{rpm}$ spindle and $340 \mathrm{~W}$ machining power connected through an automatic ATI QC 041ET tool changer. A Kistler 9257B dynamometer is mounted between the workpiece and the table, with the purpose of measuring the machining forces and torques (Nicolescu, 2005). The experimental part used was a 6082 aluminium alloy plate with 200x160 mm dimensions. The overall layout of the robotic machining cell is illustrated in Fig. 1. The purpose of the experimental setup is to determine the values of the machining parameters with respect to processed material characteristics. The structural layout of the manufacturing cell was elaborated, optimized and validated through modeling and simulation in the virtual environment offered by the ABB RobotStudio software [9]. The main goal of the simulation was to determine the required height of the robot pedestal and the optimum position of the workpiece with respect to robot's base coordinate system [10], as well as to validate the cell's workspace distribution and robot arm kinematic model [11].

The choice for mounting the Kistler force / torque sensor between the part and the workpost was made considering the fact that, using a low payload robot, it would have altered the experimental results to add more weight to the wrist [12]. Also, a fixed workpost solution was chosen for the purpose of improved stiffness [13]. The resulting robotic manufacturing cell is illustrated in Fig. 2. As already stated, the Kistler dynamometer integrated in the manufacturing cell had the purpose of measuring the machining forces at the tool-part interface. The device, having high stiffness and resolution, is capable of determining the three orthogonal values of a force by integrating four three-component sensors fitted under high preload between a baseplate and a top plate. Each sensor contains three pairs of quartz plates, one sensitive to pressure in the $\mathrm{z}$ direction and the other two responding to shear in the $\mathrm{x}-\mathrm{y}$ directions. Kistler system is shown in Fig. 3.

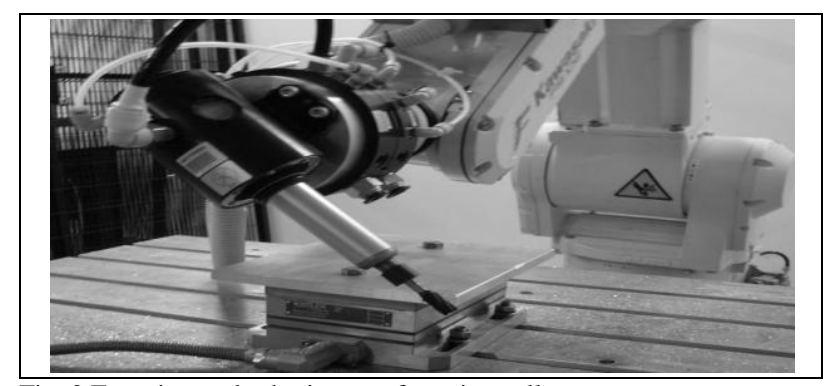

Fig. 2.Experimental robotic manufacturing cell

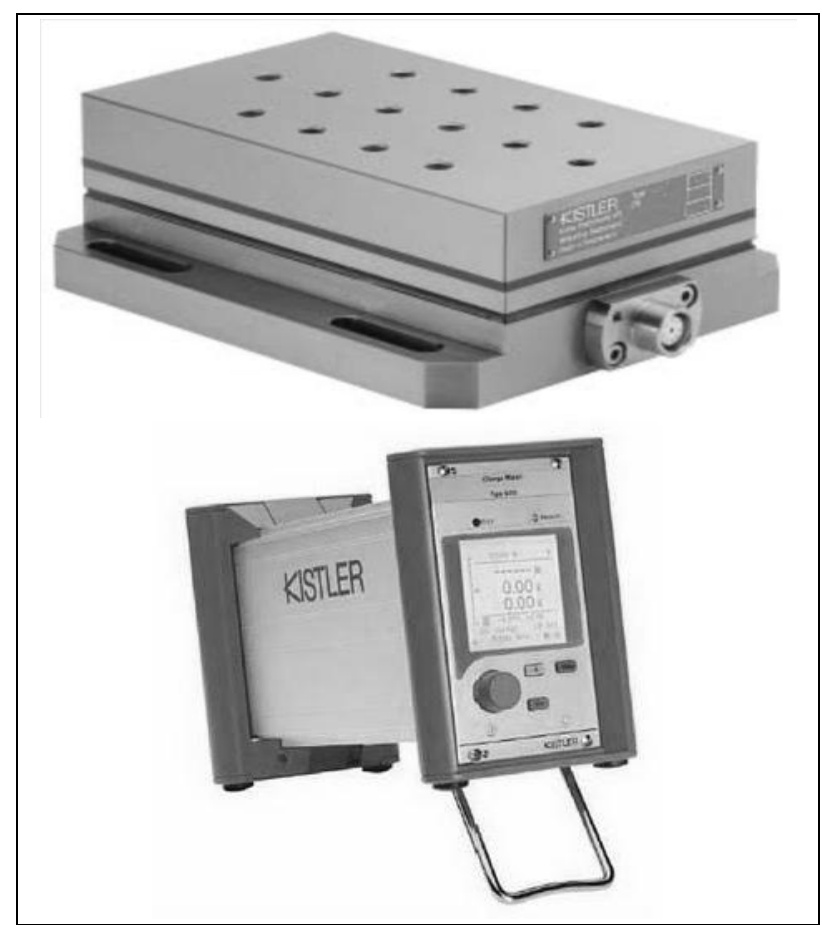

Fig. 3.The Kistler dynamometer system used for machining forces measurements

\section{EXPERIMENTAL PROCEDURE AND MEASUREMENT DATA}

In order to obtain accurate experimental data, the application programmed on the machining cell was a chamfering operation performed on the aluminium part fixed on the dynamometer top plate. The chamfering was performed at an angle of $45^{\circ}$ using a carbide tool model 9150-RC-B-24065 manufactured by ATI, featuring six teeth and a diameter of $10 \mathrm{~mm}$ dedicated for chamfering and deburring operations performed on aluminium or plastic materials.

In order to obtain an accurate range of experimental data, the robotic manufacturing cell was programmed to perform three chamfering operations. For each operation, the tool was advanced one millimeter into the part, creating a wider chamfer and increasing the amount of removed material. Thus, the cross-section of the stock varied also, from a triangle-shape during the first operation to a trapezoidal shape during the second and the third operations. To further simulate real industrial process conditions, a gap was made along the part's edge, thus simulating the cases in which the geometry of the workpiece is uneven or inconsistent. The objective of 
the experiment was to determine the behaviour of the system during the manufacturing operations expressed by machining forces values evolution. These values were collected by the PC interface of the Kistler dynamometer system and use to generate variation diagrams, as shown in Fig. 4, Fig. 5 and Fig. 6.

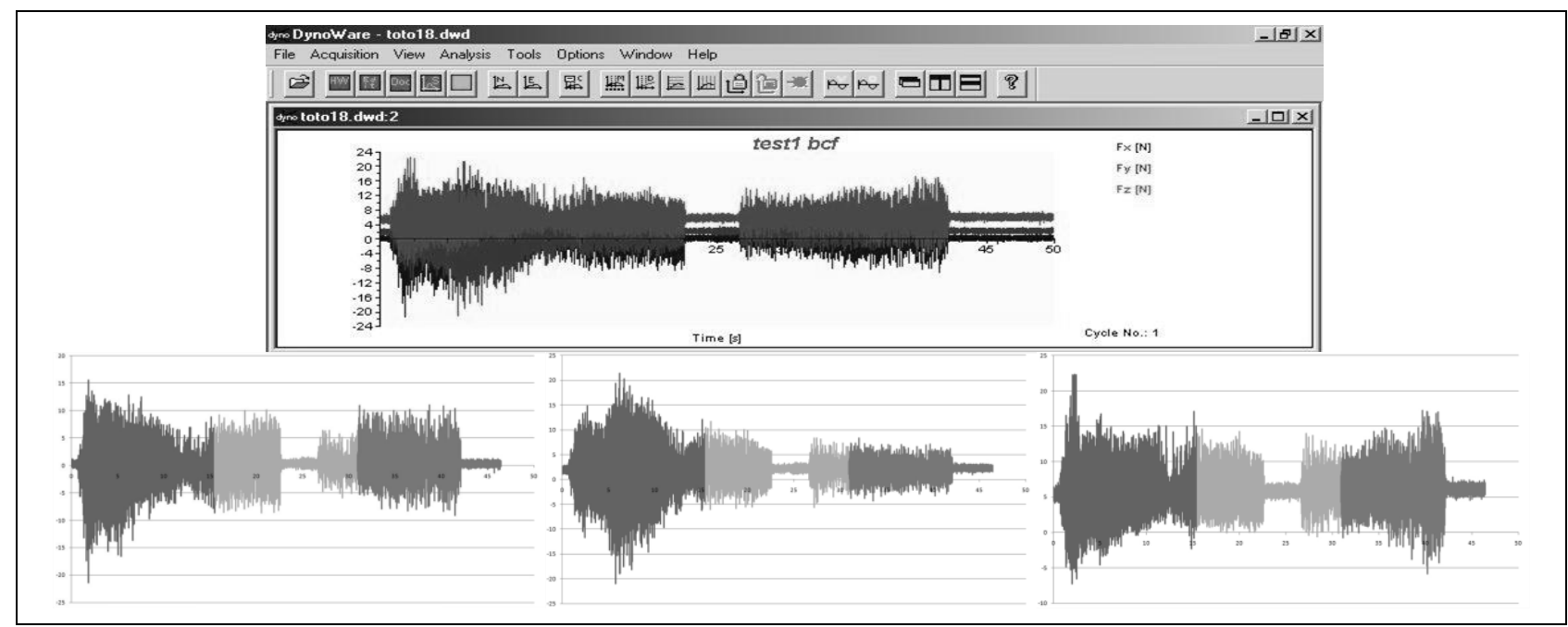

Fig. 4. Diagrams showing the evolution of the machining forces during the first chamfering operation

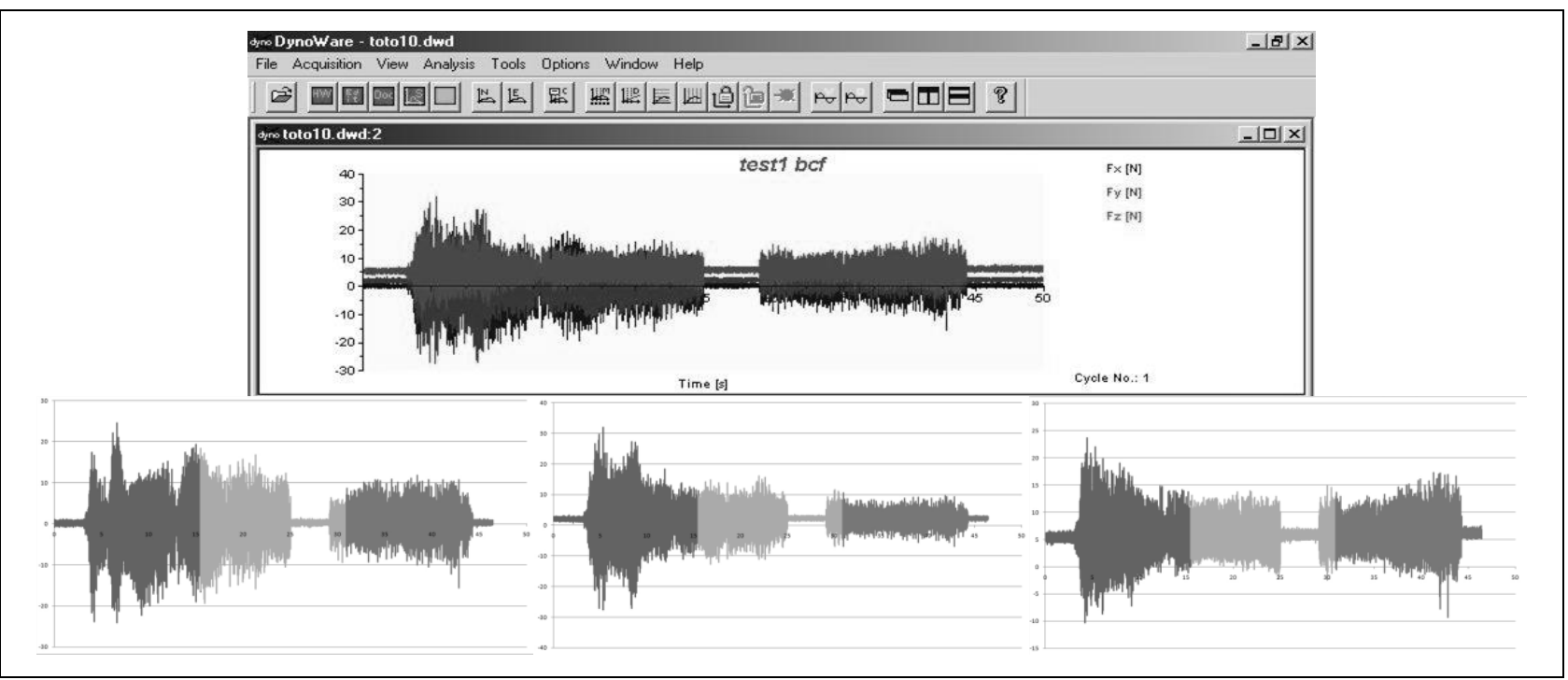

Fig. 5. Diagrams showing the evolution of the machining forces during the second chamfering operation

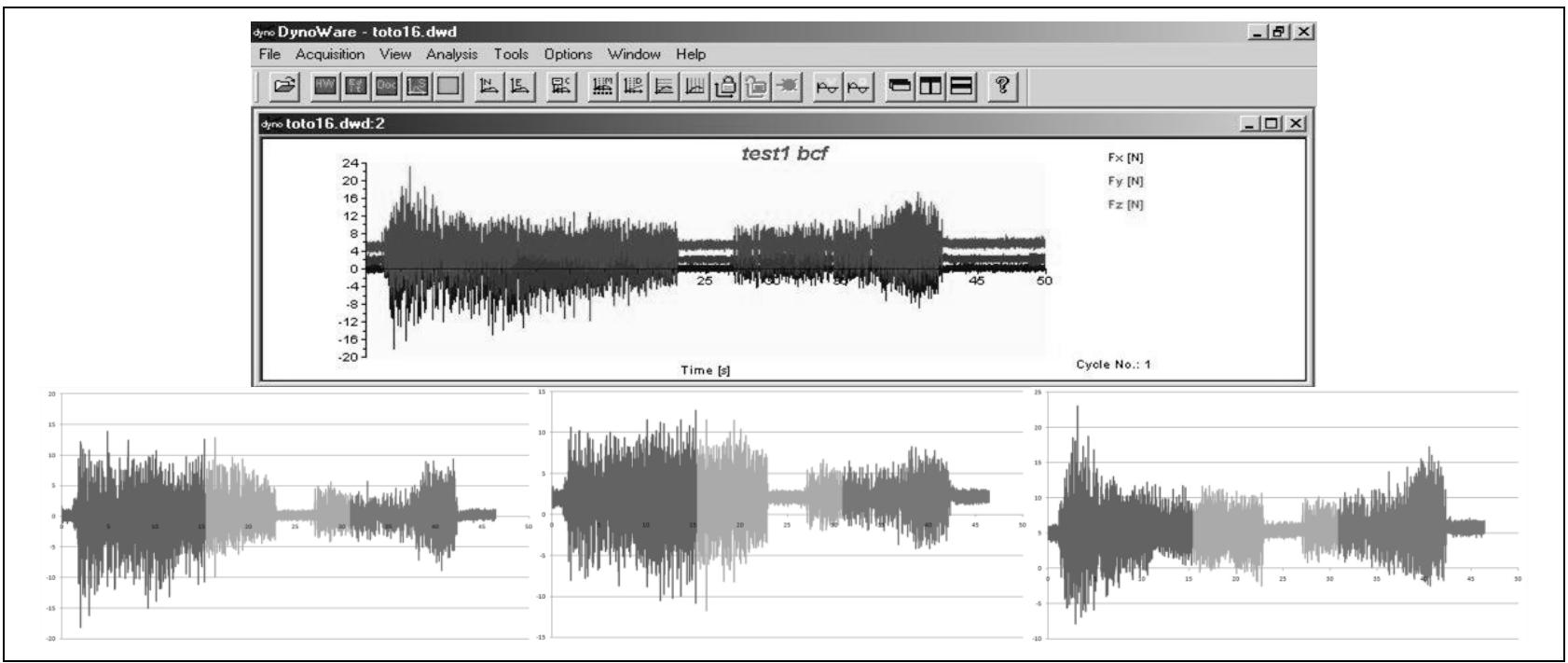

Fig. 6. Diagrams showing the evolution of the machining forces during the third chamfering operation 
Each set of diagrams shows variation of manufacturing forces values for the $\mathrm{X}, \mathrm{Y}$ and $\mathrm{Z}$ axes directions respectively, and also the superposed variation diagrams as generated by Kistler software.

\section{CONCLUSION}

The analysis of the experimental results was made taking into consideration the structure of the robotic manufacturing cell, the characteristics of the machined part and the specific parameters of the proposed chamfering operation. Taking into consideration these aspects, there can be identified four stages of the machining process:

a. the engagement stage, considered immediately following the first contact between the tool and the part;

b. the first intermediate stage, considered in the middle of the operation, before the gap in the part's edge;

c. the second intermediate stage, considered immediately after the gap in the part's edge;

d. the final stage, considered immediately before the tool's lead out.

As shown by the diagram analysis, the engagement stage was characterized by a higher level of chatter, followed by a stabilization interval. Given the fact that the cross-section size of the stock varies considerably, the chatter interval is longer in the first chamfering operation. Also, the same conclusion can be drawn regarding the engagement stage of the third chamfering operation, because the removed material volume is larger. Thus, the stabilization period following the tool's lead in depends both on the stock's volume and it's cross-section geometry. The previously described pattern appears also during the second intermediate stage, when the perturbances are much less pronounced, and during the final stage, when the chatter amplitude grows as tool approaches part edge.

As expected, the chamfering operations performed with the previously described robotic manufacturing cell do not generate high values for the machining forces measured at the tool-part interface. The illustrated diagrams show that the highest value do not exceed $25 \mathrm{~N}$. Given the fact that robot maximum payload is $10 \mathrm{~kg}$, which is equivalent to a force of 100 $\mathrm{N}$ acting at end-effector level, the main conclusion drawn from this research stage is that, for operations requiring low machining power, the forces generated at part-tool interface have a reduced influence on robot's overall performance. The experimets have shown that, as long as the machining parameters are not pushed towards the robot limits in terms of maximum payload and stiffness characteristics, the system can easily compensate for the variations in the machining forces values, even when the processed surface geometry is uneven or inconsistent.

The works presented in this paper showed that, in comparison to other factors such as robot system calibration, joint elastic displacement, tolerances of the robotic arm components, and so on, the forces at parttool interface have a relatively low influence on robot's behaviour during machining operations. When it comes to forces acting at end-effector level, analysis of the variation diagrams showed that machining forces in the discussed category of operations (requiring low machining power) only have values up to one third of the maximum robot payload.

This experimental procedure, along with the data collected from other activities showing the influence factors and error sources in robotic machining processes, is intended to form the basis of future research aimed at proposing methods for error compensation and robotic system calibration. Under these circumstances, after fully identifying and evaluating all factors that influence the absolute positioning precision and the manufacturing precision during machining operations of an articulated arm industrial robot, the next research step will be to improve robotic performance through mathemathical modeling, force control approaches and offline programming methods developed by taking into account results obtained fom present works.

\section{REFERENCES}

[1] Tolinski, M (2006). Robots Step Up to Machining, Manufacturing Engineering, vol. 137, nr. 3

[2] Bennett Brumson (2002). Take It Off: Robotic Material Removal Applications, Available from www.robotics.org/content-detail.cfm/Industrial-RoboticsNews/Take-It-Off:-Robotic-Material-RemovalApplications/content_id/1154,Accesed: 2011-05-13

[3] Elatta, A.Y., Gen, L. P., Fan, L. Z., Yu, D. \& Luo, F. (2004). An Overview of Robot Calibration, Information Technology Journal, vol. 3, ISSN 1682-6027, pp. 74-78

[4] da Motta, J. M., de Sousa, C. \& Afonso F. (2004). An Off-Line Robot Programming System Including Workcell And Robot Calibration, ABCM Symposium Series in Mechatronics, vol. 1, pp. 134-143

[5] Nicolescu, A. \& Ivan, A. (2009). Actual development status in robotic machining - a survey, 18th International Workshop on Robotics in Alpe-Adria-Danube Region (RAAD), may 2009, Brasov, Romania, ISBN 978-606-521-315-9, pp. 35

[6] Pires, J., Ramming, J., Rauch, S. \& Araújo, R. (2002) Force/torque sensing applied to industrial robotic deburring, Sensor Review, vol. 22, ISSN 0260-2288, pp.232 - 241

[7] Matsubara, A. \& Ibaraki, S. (2009). Monitoring and Control of Cutting Forces in Machining Processes: A Review, International Journal of Automation Technology, vol.3, pp. 445-456

[8] Nicolescu, A., Stanciu, M. \& Popescu, D (2004). Conceptia si exploatarea robotilor industriali, Printech Publishing House, ISBN 973-718-007-0, Bucharest, Romania

[9] Ivan, A., Nicolescu A. \& Strajescu E. (2010). Offline programming and simulation of arc welding robotic cell using RobotStudio software, Proceedings of The 21th International DAAAM Symposium, october 2010, Zadar, ISSN 1726-9679, ISBN 978-3-901509-73-5, Katalinic, B. (Ed.), pp. 0063-0064

[10] Vosniakos, G. \& Matsas, E. (2010). Improving feasibility of robotic milling through robot placement optimisation, Robotics and Computer-Integrated Manufacturing, vol. 26, ISSN 07365845, pp. 517-525

[11] Ivan, A., Nicolescu, A., Dobrescu, T. \& Strajescu, E. (2011). Development and Modeling of Robotic Manufacturing Cell for Experimental Purposes, Proceedings of The 22th International DAAAM Symposium, november 2011, Vienna, ISSN 17269679, ISBN 978-3-901509-83-4, Katalinic, B. (Ed.)

[12] Nicolescu A., Dobrescu T., Ivan A., Avram C. , Brad S., Doroftei I., Grigorescu S. (2011). Industrial robots, robotic manufacturing system and technologies, Bucharest, 2011

[13] Siciliano, B. \& Khatib, O. (2008). Springer Handbook of Robotics, Springer, ISBN: 978-3-540-23957-4, Stanford 